\title{
Fuzzy Set Approach to the Assessment of Student-Centered Learning
}

\author{
Jian Ma and Duanning Zhou
}

\begin{abstract}
Assessment of student learning is an important task in a teaching and learning process. It has a strong influence on students' approaches to learning and their outcomes. Recent development in tertiary education has shifted its emphasis from teacher-centered learning to student-centered learning. In a student-centered learning environment, criterion-referenced assessment techniques are often used in current education research and practice. However, it sometimes happens that the assessment criteria and their corresponding weights are solely determined by the lecturers in charge. This may reduce the interest of students' participation and lower the quality of their learning. This paper presents an integrated fuzzy set approach to assess the outcomes of student-centered learning. It uses fuzzy set principles to represent the imprecise concepts for subjective judgment and applies a fuzzy set method to determine the assessment criteria and their corresponding weights. Based on the commonly agreed assessment criteria, students' learning outcomes are evaluated on a fuzzy grade scale. The proposed fuzzy set approach incorporates students' opinions into assessment and allows them to have a better understanding on the assessment criteria. It aims at encouraging students to participate in the whole learning process and providing an open and fair environment for assessment.
\end{abstract}

Index Terms-Fuzzy set application, group decision support systems, project assessment, student-centered learning.

\section{INTRODUCTION}

A SSESSMENT is an important but difficult task in the whole teaching and learning process. It has a strong influence on students' approaches to learning and their learning outcomes [1], [2]. Recent development in education has shifted its emphasis from teacher-centered learning to student-centered learning [3], [4]. With the latter, students are exposed to practical problems. They often work in-groups to solve intrinsically motivating problems. Student-centered learning often takes place in the form of problem-based learning, project-based learning and lab-based learning [5]-[7]. Due to the nature of student-centered learning, criterion-referenced assessment methods are commonly used for evaluating students' outcomes [8]. The criterion-referenced assessment method directs students' attention to their performance with respect to the tasks

Manuscript received September 23, 1997; revised February 7, 2000. This work was supported in part by the Competitive Earmarked Research Grant (CERG) of Hong Kong (Project 9040375) and National Natural Science Foundation of China (NSFC, Projects 19471085 and 79600 006).

J. Ma is with the Department of Information Systems, City University of Hong Kong, Kowloon Tong, Hong Kong (e-mail: isjian@ @ityu.edu.h).

D. Zhou is with the Department of Information Systems, City University of Hong Kong, Kowloon Tong, Hong Kong, and also with the Department of Computer Sciences, Zhongshan University, China.

Publisher Item Identifier S 0018-9359(00)04190-X. they undertake [8]-[10]. However, in the current practice [8], [9] of a student-centered learning environment, the lecturers in charge are solely responsible for determining the assessment criteria and their corresponding weights. This may reduce the students' autonomy in the whole learning process and lower their learning quality. In order to encourage students to participate in the whole learning process, this paper uses the brainstorming technique [11] to allow lecturers and students to generate the basic set of assessment criteria with reference to the course objectives and the industry standards for software quality management. Based on the proposed basic set of assessment criteria, it uses the fuzzy set method for students and lecturers to vote for their preferred assessment criteria. The commonly agreed criteria are then used to evaluate the students' outcomes on a fuzzy grading scale. The proposed criterion-referenced assessment method consists of the following four steps:

Step 1) generation of the basic set of assessment criteria;

Step 2) selection of the assessment criteria from the basic set of assessment criteria;

Step 3) determination of the corresponding assessment criteria weights;

Step 4) evaluation of the students' outcomes using the agreed criteria.

The proposed assessment method aims at encouraging the students to participate in the whole teaching, learning and assessment processes. It is to provide an open and fair method for assessment.

\section{FuZZy Set ApproACH to GenerATE the AsSESSMENT CRITERIA}

Fuzzy set theory has been widely used to solve problems in the evaluation and assessment tasks [12], [13]. It is an efficient and effective method to represent the uncertainty and fuzzy terms in the assessment environments. In this paper, a fuzzy set is a class of objects with a continuum of membership grades. A membership function, which assigns to each object a grade of membership, is associated with the fuzzy set. Usually, the membership grades are in $[0,1]$. When the grade of membership for an object is one, it means that the object is absolutely in that set. When the grade of membership is zero, it means that the object is absolutely not in that set. Borderline cases are assigned to the values between zero and one. Precise membership grades do not convey any absolute significance. They are context-dependent and can be subjectively assessed. Fuzzy set method has been 


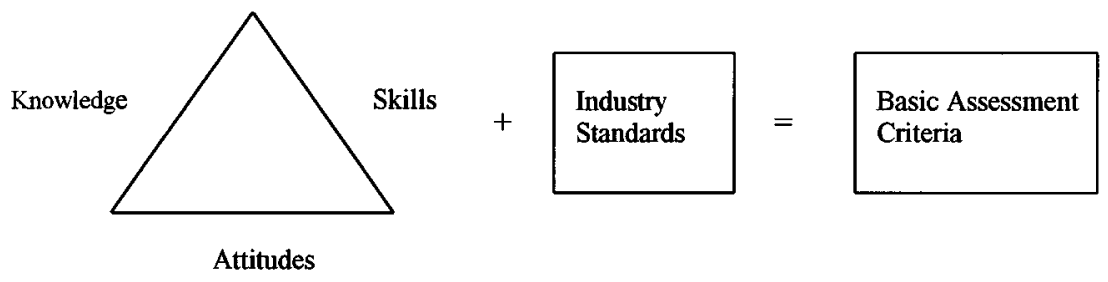

Fig. 1. Content of basic assessment criteria.

applied in the assessment process [14], [15]. Echauz and Vachtsevanos [14] presented a fuzzy grading method that utilizes students' and instructor's performance measure to produce a 'fair' mark distribution. Rantjt [15] described a fuzzy set to evaluate students' answer scripts.

Let $U$ be a classical set of objects, called the universe, where $U=\{x\}$, whose generic elements are denoted by $x$. A fuzzy set $A$ is characterized by a membership function $\mu_{A}(x)$ which associates each element in $U$ with a real value between $[0,1]$. The fuzzy set $A$ is usually denoted by a set of pairs $A=\left\{\left(x, \mu_{A}(x)\right), x \in U, \mu_{A}(x) \in[u, 1]\right\}$. When $U$ is a finite set, i.e., $\left\{x_{1}, \ldots, x_{n}\right\}$, the fuzzy set on $U$ can be represented as: $A=\sum_{i=1}^{n} x_{i} / \mu_{A}\left(x_{i}\right)$. If there is a natural ordering of the elements in the universe $U$, one can simply use the vector $\left(\mu_{A}\left(x_{1}\right), \ldots, \mu_{A}\left(x_{n}\right)\right)$ of the membership degree to represent the fuzzy set $A$. All fuzzy sets on $U$ is denoted as $\Gamma(U)$. The cardinality of fuzzy set $A$ is defined by the sum of the membership degrees: $\operatorname{Card}(A)=\sum_{i=1}^{n} \mu_{A}\left(x_{i}\right)$ [13].

Classification is one of the major applications in fuzzy set research [13]. In this paper, let $U$ be a universe, $A_{1}, \ldots, A_{n}$ be fuzzy sets in $U$, i.e., $A_{1}, \ldots, A_{n} \in \Gamma(U)$, and $u \in U$ be an object. If $A_{i}$ satisfies $\mu_{A_{i}}(u)=\max \left\{\mu_{A_{1}}(u), \ldots, \mu_{A_{n}}(u)\right\}, u$ can be classified into $A_{i}$.

Let $X, Y$ be the universes and $\Gamma(Y)$ be the set of all fuzzy sets in $Y$, then $A=\left\{\left((x, y), \mu_{A}(x, y)\right), \mu_{A}(x, y) \in[0,1],(x, y) \subseteq\right.$ $X \times Y\}$ is called a fuzzy relation on $X \times Y . f: X \rightarrow \Gamma(Y)$ is called a fuzzy function from $X$ to $Y$. The fuzzy functions and fuzzy relations are inter-related and both of them can be represented by the membership matrix. The composition of two fuzzy relations $P(X, Y)$ and $Q(X, Y)$ can be defined in terms of an operation on the membership matrix $P$ and $Q$ that resembles matrix multiplication. This operation involves exactly the same combinations of matrix entries like the regular matrix multiplication. However, the multiplication and additions applied to these combinations in the matrix multiplication are replaced with other operations. These alternative operations represent, in the given context, the corresponding operations of fuzzy set intersection and union. In the Max-Min composition, for example, the multiplication and addition are replaced with the Min and Max operations, respectively. The composition result on $P(X, Y)$ and $Q(X, Y)$ is a fuzzy relation $R(X, Y)$, which can be denoted as $R=P \circ Q$.

A brainstorming technique is used to generate the basic set of assessment criteria, where the lecturer can lead the students to study the course objectives (e.g., major course requirements in knowledge, skills and attitudes) and relevant international standards for software quality management. They work collabora- tively to propose a basic set of assessment criteria, denoted as $C=\left\{c_{1}, \ldots, c_{m}\right\}$. The content of the criteria is shown in Fig. 1 .

A fuzzy voting method is used for students and lecturers to rank the criteria from the basic set of assessment criteria. Where every participant is asked to give preference information on each criterion. A fuzzy value between $[0,1]$ is assigned by the participant to represent his/her preference over the criteria. The larger the fuzzy value is assigned, the more important that criterion is. Finally a fuzzy set is obtained with values assigned to each criterion.

Normally, there are $k$ decision makers (DM's), i.e., students and lecturers, denoted as $P=\left\{p_{1}, \ldots, p_{k}\right\}$, participating in the assessment process. Each DM is to use a fuzzy value ranging between $[0,1]$ to rank the importance of the assessment criteria. Thus, the ranking preference by the DM $p_{i}$ is represented in the vector $V_{i}=\left(v_{i 1}, \ldots, v_{i m}\right)$, where $v_{i j}$ is the preference value that DM $p_{i}$ gives to the criterion $c_{j}$, and $v_{i j} \in$ $[0,1],(i=1, \ldots, k ; j=1, \ldots, m)$. According to psychologist Miller's famous theory, whereby humans can only grasp accurately about seven different chunks of information [16], the optimal number of assessment criteria is $7 \pm 2$, i.e., the cardinality of fuzzy set $V_{i}$ should be less than or equal to 9 . Thus, we have, $\operatorname{Card}\left(V_{i}\right)=\sum_{j=1}^{m} v_{i j} \leq 9$.

When $k$ DM's give their preference on $m$ assessment criteria, a fuzzy matrix is formed, denoted as $V=\left(v_{i j}\right)(i=1, \ldots, k ; j=1, \ldots, m)$. Current research on assessment [7], [8] shows that lecturers should play a major role in determining the assessment criteria for assessing students' group projects. Lecturers generally have clear expectations on the course requirements and experience, they should have the priority in expressing their opinions on the assessment criteria. This can be realized by assigning different rates (usually determined by the lecturers), denoted as $Q=\left\{q_{1}, \ldots, q_{k}\right\}$, to the individual DM's, where $\sum_{i=1}^{k} q_{i}=1$. For example, if there are one lecturer $\left(p_{1}\right)$, two tutors $\left(p_{2}, p_{3}\right)$, and eight students ( $p_{4}$ to $p_{11}$ ) involved in the selection process $(k=11)$, then $0.4,0.1$, and 0.05 could be assigned to the lecturer, tutors and students, respectively, where $Q=$ $(0.4,0.1,0.1,0.05,0.05,0.05,0.05,0.05,0.05,0.05,0.05)$.

Thus, the final assessment criteria can be obtained by fuzzy set composition

$$
\begin{aligned}
V^{\prime} & =Q \circ V=\left(q_{1}, q_{2}, \ldots, q_{k}\right) \circ\left[\begin{array}{c}
v_{11}, v_{12}, \ldots, v_{1 m} \\
v_{21}, v_{22}, \ldots, v_{2 m} \\
\ldots \\
v_{k 1}, v_{k 2}, \ldots, v_{k m}
\end{array}\right] \\
& =\left(v_{1}^{\prime}, v_{2}^{\prime}, \ldots, v_{m}^{\prime}\right)
\end{aligned}
$$


where $v_{i}^{\prime}=\left(q_{1} \bullet v_{1 i}\right) \oplus\left(q_{2} \bullet v_{2 i}\right) \oplus \cdots \oplus\left(q_{k} \bullet v_{k i}\right)$ and "•", " $\oplus$ " are defined as

$$
\begin{aligned}
& \text { algebraic product, } a \bullet b \text { : } c=a b \\
& \text { bounded sum, } a \oplus b: c=a \oplus b=\min \{1, a+b\} .
\end{aligned}
$$

In (1), the vector $V^{\prime}$ can be sorted in a descending order, with around top seven most popular elements being selected. The corresponding criteria are chosen as the final assessment criteria set, denoted as $C=\left\{c_{1}, \ldots, c_{n}\right\}$.

Different weights should be assigned to the assessment criteria with reference to their importance. The quantified judgments on pairs of criteria $c_{i}, c_{j}$, are represented by an $n$-by- $n$ matrix $A=\left(a_{i j}\right)(i, j=1, \ldots, n)$. The matrix is a reciprocal matrix $\left(a_{i j}=1 / a_{j i}\right)$ with all $a_{i j}$ being positive. The ratio $a_{i j}$ represents the relative importance of $c_{i}$ over $c_{j}$. The comparison scale ranges from 1 to 9 , each representing the concepts of: 1: equally important; 3: weakly more important; 5: strongly more important; 7: demonstratively more important; 9: absolutely more important; 2, 4, 6, and 8 are intermediate values between adjacent judgments [17]. For example, $a_{i j}=5$ means $c_{i}$ is strongly more important than $c_{j}$. Thus, the weights $W=\left(w_{1}, \ldots, w_{n}\right)$ on the criteria $C=\left\{c_{1}, \ldots, c_{n}\right\}$ can be computed according to the paired comparison matrix [17] contributed by the students and lecturers in the class.

\section{FUZZY GRADING SYSTEM}

In a student-centered learning environment, students are encouraged to play an active role in evaluating their learning outcomes. Thus, the proposed assessment method should also support the peer evaluation [7], [8]. Assume there are $t$ participants, i.e., lecturers and students, denoted as $P=\left\{p_{1}, \ldots, p_{t}\right\}$, involved in the evaluation process. Different rates are assigned to the participants, respectively, denoted as $R=\left\{r_{1}, \ldots, r_{t}\right\}$, where $\sum_{i=1}^{t} r_{i}=1$.

In this paper, students are asked to express the evaluation results using a 100-point scale. For example, 80 indicates the learning outcomes to be $80 \%$ satisfactory according to the commonly agreed criteria. Thus, the evaluation results given by participant $p_{i}$ form a vector $M_{i}=\left\{m_{i 1}, \ldots, m_{i n}\right\}$, where each $m_{i j} \in[0,100],(i=1, \ldots, t ; j=1, \ldots, n)$, is the assigned mark with reference to the criterion $c_{j}$.

For $t$ participants involved in the evaluation process, an evaluation matrix $M=\left(m_{i j}\right)(i=l, \ldots, t ; j=l, \ldots, n)$ is formed. Thus, the evaluation results can be obtained by

$$
\begin{aligned}
M^{\prime} & =R * M=\left(r_{1}, r_{2}, \ldots, r_{t}\right)\left[\begin{array}{c}
m_{11}, m_{12}, \ldots, m_{1 n} \\
m_{21}, m_{22}, \ldots, m_{2 n} \\
\ldots ., \\
m_{t 1}, m_{t 2}, \ldots, m_{t n}
\end{array}\right] \\
& =\left(m_{1}^{\prime}, m_{2}^{\prime}, \ldots, m_{n}^{\prime}\right)
\end{aligned}
$$

where $m_{i}^{\prime}=r_{1} \times m_{1 i}+r_{2} \times m_{2 i}+\cdots+r_{t} \times m_{t i}$ and " $\times$ ", "+" are the normal mathematical operators for multiplication and addition, respectively.

Letter grades are always needed which reflect the students' performance in the University. Thus, a membership function needs to be defined which transforms a mark grade into a letter grade, namely, $G=\left\{g_{1}, \ldots, g_{d}\right\}$. The fuzzy letter grade $g_{i}$ can be represented by the membership function $\mu_{g_{i}}(i=1, \ldots, d)$. Hence, given a 100-point scale mark $m^{\prime}, \mu_{g_{g}}\left(m^{\prime}\right)$ represents the degree that the mark $m^{\prime}$ belongs to the letter grade $g_{i}$.

A fuzzy mapping can be defined which combines the membership functions together to form a comprehensive letter grade

$$
\begin{aligned}
f: C & \rightarrow \Gamma(G) \\
c_{i} & \rightarrow\left(\mu_{g_{1}}\left(m_{i}^{\prime}\right), \mu_{g_{2}}\left(m_{i}^{\prime}\right), \ldots, \mu_{g_{d}}\left(m_{i}^{\prime}\right)\right.
\end{aligned}
$$

where $m_{i}^{\prime}$ is the mark given to $c_{i}, m_{i}^{\prime} \in[0,100], i=1, \ldots, n$. With the membership functions $\mu_{g_{1}}, \ldots, \mu_{g_{d}}$, the fuzzy mapping $f$ gives each mark $m_{i}^{\prime}$ the degree which belongs to the letter grades $g_{1}, \ldots, g_{d}$, respectively.

For simplicity, use $\left(e_{i_{1}}, \ldots, e_{i_{d}}\right)$ to represent $\left(\mu_{g_{1}}\left(m_{i}^{\prime}\right), \ldots, \mu_{g_{d}}\left(m_{i}^{\prime}\right)\right)$. Thus, the fuzzy evaluation matrix $E$ is expressed as

$$
\begin{gathered}
E=\left[\begin{array}{c}
e_{11}, e_{12}, \ldots, e_{1 d} \\
e_{21}, e_{22}, \ldots, e_{2 d} \\
\ldots \\
e_{n 1}, e_{n 2}, \cdots, e_{n d}
\end{array}\right]=\left[e_{i j}\right] \\
(i=1,2, \ldots, n ; j=1,2, \ldots, d) .
\end{gathered}
$$

Thus, the final letter grade of the students' learning outcomes can be determined by the evaluation result vector $Y$

$$
\begin{aligned}
Y & =W \circ E=\left(w_{1}, w_{2}, \ldots, w_{n}\right) \circ\left[\begin{array}{c}
e_{11}, e_{12}, \ldots, e_{1 d} \\
e_{21}, e_{22}, \ldots, e_{2 d} \\
\ldots \\
e_{n 1}, e_{n 2}, \ldots, e_{n d}
\end{array}\right] \\
& =\left(y_{i}\right) \quad i=1,2, \ldots, d
\end{aligned}
$$

where $y_{i}=\left(w_{1} \bullet e_{1 i}\right) \oplus\left(w_{2} \bullet e_{2 i}\right) \oplus \cdots \oplus\left(w_{n} \bullet e_{n i}\right)$.

According to the principles of fuzzy classification [13], we have $y_{i}=\max \left(y_{1}, \ldots, y_{d}\right)$. Thus, the corresponding letter grade $g_{i}$ is the students' final grade.

\section{Application of the Proposed Assessment Method}

A group decision support system (GDSS) [18] has been developed to facilitate the assessment process and to automate the computation of fuzzy matrix operations. The proposed assessment method and the GDSS have been used in assessing the students' learning outcomes in a problem-based learning task in the Department of Information Systems at the City University of Hong Kong [19], [20].

Past experience with a second year undergraduate course on distributed and client/server systems first exposed 213 students to a practical problem. They were urged to analyze the requirements from different user groups and to develop a distributed database system for the management and support of research projects in the University.

At beginning of the semester, the lecturer led the students to discuss the course objectives and the software quality standards such as ISO-9126 [21]. Students and lecturers then sat down together and used the GDSS system to brainstorm the possible assessment criteria set. The resultant basic set of assessment criteria included: $C=\{$ functionality, reliability, usability, efficiency, maintainability, portability, reusability $\}$. 


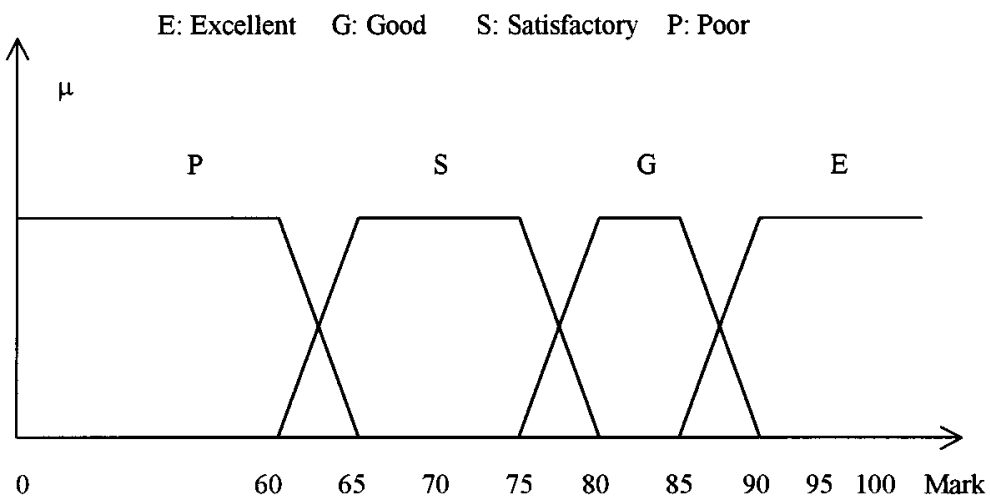

Fig. 2. Relationships between 100-point scale marks and fuzzy membership functions.

Based on the basic set of assessment criteria, students and lecturers then used the fuzzy voting method to agree on the final assessment criteria and on their corresponding weights. They were to rank the criteria using a fuzzy value in $[0,1]$. For example, if five suitable criteria from the above seven criteria were selected, a sample ranking vector from a student was given as

$$
(1,1,0.8,1,0.6,0.6,0) \text {. }
$$

The ranking order from the whole class then formed an evaluation matrix. By multiplying the voting weights with the evaluation matrix in the GDSS, the selection vector $V=(0.97,0.972,0.98,0.81,0.832,0.32,0.182)$ was obtained. Ranking the vector in descending order, the top five criteria could be selected by the GDSS as the agreed final assessment criteria, i.e., $C=\{$ functionality, reliability, usability, efficiency, maintainability\}.

The lecturers and students were asked to compare the relative importance of every two criteria so as to form a paired comparison matrix of the assessment criteria. A sample paired comparison matrix from a student was given as follows:

$$
\left[\begin{array}{ccccc}
1 & 1 & 1 / 3 & 4 & 5 \\
1 & 1 & 1 / 3 & 5 & 5 \\
3 & 3 & 1 & 7 & 7 \\
1 / 4 & 1 / 5 & 1 / 7 & 1 & 1 / 3 \\
1 / 5 & 1 / 5 & 1 / 7 & 3 & 1
\end{array}\right]
$$

The element of the matrix $a_{i j}$ represented the relative importance of criterion $c_{i}$ over criterion $c_{j}$. For example, the first row of the matrix meant that functionality was equally important as itself, functionality was equally important as reliability, usability was weakly more important than functionality, functionality was more important than efficiency, and functionality was strongly more important than maintainability.

By calculating the normalized principle eigenvector of every participant's matrix, the criteria's weights, $W=(0.205502,0.214881,0.475251,0.042013,0.062352)$ were computed by the GDSS.

As agreed, the four scale letter grades were used in the assessment. They included: $g_{1}=E$ : Excellent, $g_{2}=G$ : Good,
$g_{3}=S$ : Satisfactory, $g_{4}=P$ : Poor. Their corresponding membership functions were defined as

$$
\begin{aligned}
& \mu_{E}(u)= \begin{cases}0 & 0 \leq u \leq 85 \\
(u-85) / 5 & 85<u \leq 90 \\
1 & 90<u \leq 100\end{cases} \\
& \mu_{G}(u)= \begin{cases}0 & 0 \leq u \leq 75 \\
(u-75) / 5 & 75<u \leq 80 \\
1 & 80<u \leq 85 \\
(90-u) / 5 & 85<u \leq 90 \\
0 & 90<u \leq 100\end{cases} \\
& \mu_{S}(u)= \begin{cases}0 & 0 \leq u \leq 60 \\
(u-60) / 5 & 60<u \leq 65 \\
1 & 65<u \leq 75 \\
(80-u) / 5 & 75<u \leq 80 \\
0 & 80<u \leq 100\end{cases} \\
& \mu_{P}(u)=\left\{\begin{array}{ll}
1 & 0 \leq u \leq 60 \\
(65-u) / 5 & 60<u \leq 65 \\
0 & 65<u \leq 100
\end{array} .\right.
\end{aligned}
$$

If we let $E, G, S$, and $P$ to stand for Excellent, Good, Satisfactory and Poor, respectively, the relationships between 100-point scale marks and fuzzy membership functions can be seen in Fig. 2.

According to the membership functions in (6)-(9) and Fig. 2, if a student received a score of 78, his/her corresponding membership degrees are $\mu_{E}(78)=0, \mu_{G}(78)=0.6, \mu_{S}(78)=$ $0.4, \mu_{P}(78)=0$. This means that the student has 0 degree belongs to the grade $E, 0.6$ degree belongs to the grade $G, 0.4$ degree belongs to the grade $S$, and 0 degree belongs to the grade P.)

The students' learning outcomes were evaluated by their peers and the lecturer. A sample evaluation matrix $E$ of a student was given as follows:

$$
E=\left[\begin{array}{lccc}
0.06 & 0.8 & 0.14 & 0 \\
0 & 0.74 & 0.26 & 0 \\
0 & 0.94 & 0.06 & 0 \\
0 & 0 & 1 & 0 \\
0 & 0 & 0.3 & 0.7
\end{array}\right]
$$


According to (5), the final letter grade of the students' learning outcomes could be determined by

$$
\begin{aligned}
Y= & W \circ E \\
= & (0.205502,0.214881,0.475251,0.042013,0.062352) \\
& \circ\left[\begin{array}{cccc}
0.06 & 0.8 & 0.14 & 0 \\
0 & 0.74 & 0.26 & 0 \\
0 & 0.94 & 0.06 & 0 \\
0 & 0 & 1 & 0 \\
0 & 0 & 0.3 & 0.7
\end{array}\right] \\
= & (0.012330,0.770150,0.173873,0.043647) .
\end{aligned}
$$

The maximum value in $Y$ was $y_{2}$. Thus, the final grade of the student was $g_{2}=G$ (Good).

Agreements on the set of assessment criteria was reached in the first two weeks and the project deliverables were graded in week 13 of the Semester. Empirical studies were conducted by separating students into the experimental groups (GDSS support) and control groups (non-GDSS support). The experimental results showed [22], [23] that the proposed assessment method and the GDSS enhanced students' understanding on the assessment requirements. Comparison of project deliverable scores also revealed that the average project scores of the experimental groups were higher than that of the control groups. Also the experimental groups had higher score in deep approaches to learning that that of the control groups as measured by the study process questionnaire (SPQ).

\section{SUMMARY}

This paper presents a fuzzy set approach to assess the outcomes of student-centered learning. The proposed method uses a fuzzy set method for determining the assessment criteria and their corresponding weights. The commonly agreed criteria are then used to evaluate the students' learning outcomes. A GDSS system has been developed to support the assessment method and its process. The proposed assessment method and GDSS system encourages the students to participate in the evaluation of their learning. It also provides a convenient method for lecturers and students to express their opinions and to agree on the assessment criteria and the evaluation marks in fuzzy terms that are commonly used in their assessment tasks.

\section{ACKNOWLEDGMENT}

The authors thank three anonymous referees who made the useful comments and suggestions on the content and presentation of the paper.

\section{REFERENCES}

[1] J. Heywood, Assessment in Higher Education, 2nd ed. New York: Wiley, 1989.

[2] L. Dahlgren, "Outcomes of learning," in The Experience of Learning, F. Marton, D. J. Hounsell, and N. J. Entwistle, Eds. Edinburgh, U.K.: Scottish Academic, 1984, pp. 1-18.

[3] Y. W. Leung, "Least-square-error estimate of individual contribution in group project," IEEE Trans. Educ., vol. 41, pp. 282-285, Nov. 1998.

[4] D. A. Norman and J. C. Spohrer, "Learner-centered education," Commun. ACM, vol. 39, no. 4, pp. 24-27, 1996.
[5] J. Ma, "Problem-based learning with database systems," Computers and Education, vol. 22, no. 3, pp. 257-263, 1994

[6] D. Boud, Ed., Problem-Based Learning in Education for the Professions. Sydney, Australia: Higher Education Research and Development Society of Australia, 1985.

[7] W. Cheng and M. Warren, "Peer and teacher assessment of the oral and written tasks of a group project," Assessment and Evaluation in Higher Education, vol. 24, no. 3, pp. 301-314, 1999.

[8] F. Lopez-Real and Y. P. Chan, "Peer assessment of a group project in a primary mathematics education course," Assessment and Evaluation in Higher Education, vol. 24, no. 1, pp. 67-73, 1999.

[9] G. Gibbs, S. Habeshaw, and T. Habeshaw, 53 Interesting Ways to Assess Your Students. Bristol, Melbourne, Australia: Technical and Educational Services, 1996

[10] R. A. Berk, Ed., A Guide to Criterion-Referenced Test Construction. Baltimore, MD: The Johns Hopkins Press, 1984.

[11] C. L. Hwang and M. J. Lin, Group Decision Making under Multiple Criteria: Methods and Applications. New York: Springer-Verlag, 1987.

[12] S. Feng and L. D. Xu, "Decision support for fuzzy comprehensive evaluation of urban development," Fuzzy Sets Syst., vol. 105, pp. 1-12, 1999.

[13] G. J. Klirm and B. Yuan, Fuzzy Sets and Fuzzy Logic: Theory and Applications. Englewood Cliffs, NJ: Prentice-Hall, 1995.

[14] J. R. Echauz and G. J. Vachtsevanos, "Fuzzy grading system," IEEE Trans. Educ., vol. 38, no. 2, pp. 158-164, 1995.

[15] B. Ranjit, "An application of fuzzy set in students' evaluation," Fuzzy Sets and Systems, vol. 74, pp. 187-194, 1995.

[16] G. A. Miller, "The magical number seven, plus or minus two: Some limits on our capacity for processing information," Psychol. Rev., vol. 63, pp. 81-97, 1956.

[17] T. L. Saaty, Fundamentals of Decision Making and Priority Theory with the Analytic Hierarchy Process: RWS, 1994.

[18] J. Ma, "Group decision support system for assessment of problem-based learning," IEEE Trans. Educ., vol. 39, pp. 388-393, Aug. 1996.

[19] D. Zhou, J. Ma, R. C. W. Kwok, and Q. Tian, "Group decision support system for project assessment based on fuzzy set theory," presented at the Proc. 32nd Hawaii Int. Conf. System Sciences (HICSS-32), Honolulu, HI, Jan. 1999.

[20] R. Kwok and J. Ma, "Use of group support system for collaborative assessment," Comput. Educ. Int. J., vol. 32, pp. 109-125, 1999.

[21] Software Product Evaluation: Quality Characteristics and Guidelines for Their Use, 1991.

[22] R. Kwok, J. Ma, D. Vogel, and D. Zhou, Collaborative Assessment in Education: Application of Fuzzy Sets, Working paper of IS Department, City University of Hong Kong, 1999.

[23] R. Kwok and J. Ma, GSS: Collaborative Technology for Assessment in IS Education, Working paper of IS Department, City University of Hong Kong, 1999.

Jian Ma received the Dr. Eng. degree in computer science from the Asian Institute of Technology, Bangkok, Thailand, in 1991.

$\mathrm{He}$ is currently an Associate Professor in the Department of Information Systems at the City University of Hong Kong. He was a Lecturer in the School of Computer Science and Engineering at the University of New South Wales, Australia, before joining the City University in 1993. His current research areas include web-based decision support systems, object-oriented and componentbased methods for information systems development, and use of IT in business education. His past research has been published in IEEE TRANSACTIONS ON Education, IEEE Transactions on Systems, Man, AND Cybernetics, $D e$ cision Support Systems, and Computers and Education.

Duanning Zhou received the B.Sc. degree in mathematics from Jiangxi University, Nanchang, China, in 1984, the M.Eng. degree in computer science from Zhongshan (Sun Yasen) University, Guangzhou, China, in 1990, and the Ph.D. degree in information systems from the City University of Hong Kong in 2000 .

He was a Lecturer in the Department of Computer Science, Zhongshan University, before joining the Department of Information Systems at City University of Hong Kong for his Ph.D. studies in 1996. His research interests are in the areas of web-based decision support systems, fuzzy logic, software agent-based systems, and electronic commerce. 